\title{
Compact Linear Collider drive beam phase stabilization simulations
}

\author{
A. Gerbershagen, ${ }^{1,2, *}$ D. Schulte, ${ }^{1}$ and P. N. Burrows ${ }^{2}$ \\ ${ }^{1}$ CERN, Geneva 1217, Switzerland \\ ${ }^{2} J A I$, University of Oxford, Oxford OXI 3RH, United Kingdom \\ (Received 5 February 2013; revised manuscript received 18 June 2014; published 13 April 2015)

\begin{abstract}
The drive beam phase stability is one of the critical feasibility issues of the Compact Linear Collider (CLIC) project. This paper presents a step-by-step analysis of the error propagation through the CLIC drive beam complex using realistic rf potential and beam loading amplitude functions for the drive and main beam accelerating structures. The impact of planned stabilization systems for drive beam bunch charge and longitudinal phase is simulated and the optimal specifications for such systems are calculated and discussed.
\end{abstract}

DOI: 10.1103/PhysRevSTAB.18.041003

PACS numbers: 29.20.Ej

\section{INTRODUCTION}

The Compact Linear Collider (CLIC) [1] is a proposed $e^{+} e^{-}$collider being developed at CERN. It is designed to accelerate the electron and positron beams with an acceleration gradient of $100 \mathrm{MV} / \mathrm{m}$ and collide them at the nominal center-of-mass energy of $3 \mathrm{TeV}$.

The rf wave needed for such acceleration cannot be provided cost effectively with klystrons, hence a novel twobeam solution has been chosen. The so-called drive beam with low energy and high current is used to deliver rf power for the acceleration of the high energy, low current main beams, which are brought into collision (Fig. 1). Both the drive and the main beams are generated in a central injector complex and are transported along the linac to the corresponding turnarounds. Delivering the rf power via the drive beam also gives the advantage of lower power losses in transporting the beam pulses and ability to compress them to higher bunch frequency.

This paper describes in detail the propagation of the drive beam errors, in particular the phase error, and their impact on the main beam and hence on luminosity. Also, the examination of the possibility of reducing the drive beam phase error with a feed-forward correction by a factor of 12.5 is presented, which is required to satisfy the drive beam phase tolerance requirement (see the following section).

The current study uses the results of the previous simulation work for the drive beam [2] and main beam [3] rf filling and beam loading functions as well as the beam propagation through the bunch compression chicane [4]. It merges these results with the simulation of the beam

*alexander.gerbershagen@ cern.ch

Published by the American Physical Society under the terms of the Creative Commons Attribution 3.0 License. Further distribution of this work must maintain attribution to the author(s) and the published article's title, journal citation, and DOI. propagation through the drive beam recombination scheme, analyzing it in full complexity, which is discussed in detail in Sec. III A. The aim of the study is to confirm the assumptions used for the definition of CLIC drive beam tolerances and to set the specifications for the potential drive beam bunch charge feedback and phase feed-forward systems.

For the purpose of this analysis a dedicated software tool has been developed. Figure 2 illustrates in a simplified diagram the operation of the tool. The tool simulates the errors in initial parameters such as beam charge and phase at the source, the phase and amplitude of the drive beam accelerating structures etc. It allows the tracking of the error propagation and transformation along the different sections of the drive beam complex. Finally, it performs the calculation of the impact of these errors on the main beam and the prediction of the total resulting luminosity loss of CLIC. The detailed description of the functionality and the structure of the software simulation tool can be found in [5].

The paper is structured in the following way: in Sec. II propagation of the errors through the drive beam accelerator is described. In Sec. III the filtering of the errors by the recombination scheme as well as by the main beam accelerating structures is described. Section IV discusses the impact of the bunch charge feedback and phase feedforward correction systems. Section V summarizes the results, explains the significance of the study and presents an outlook for future simulations and experiments.

\section{A. CLIC drive beam tolerances}

The design beam spot size at the interaction point of the CLIC main beams is $40 \mathrm{~nm}$ horizontally and $1 \mathrm{~nm}$ vertically [6]. These values are very challenging to achieve and require a high degree of stabilization of many parameters of the main beam, including the energy spread. Jitters of the drive beam phase and current lead to jitters of the accelerating rf wave for the main beam. These lead to main 


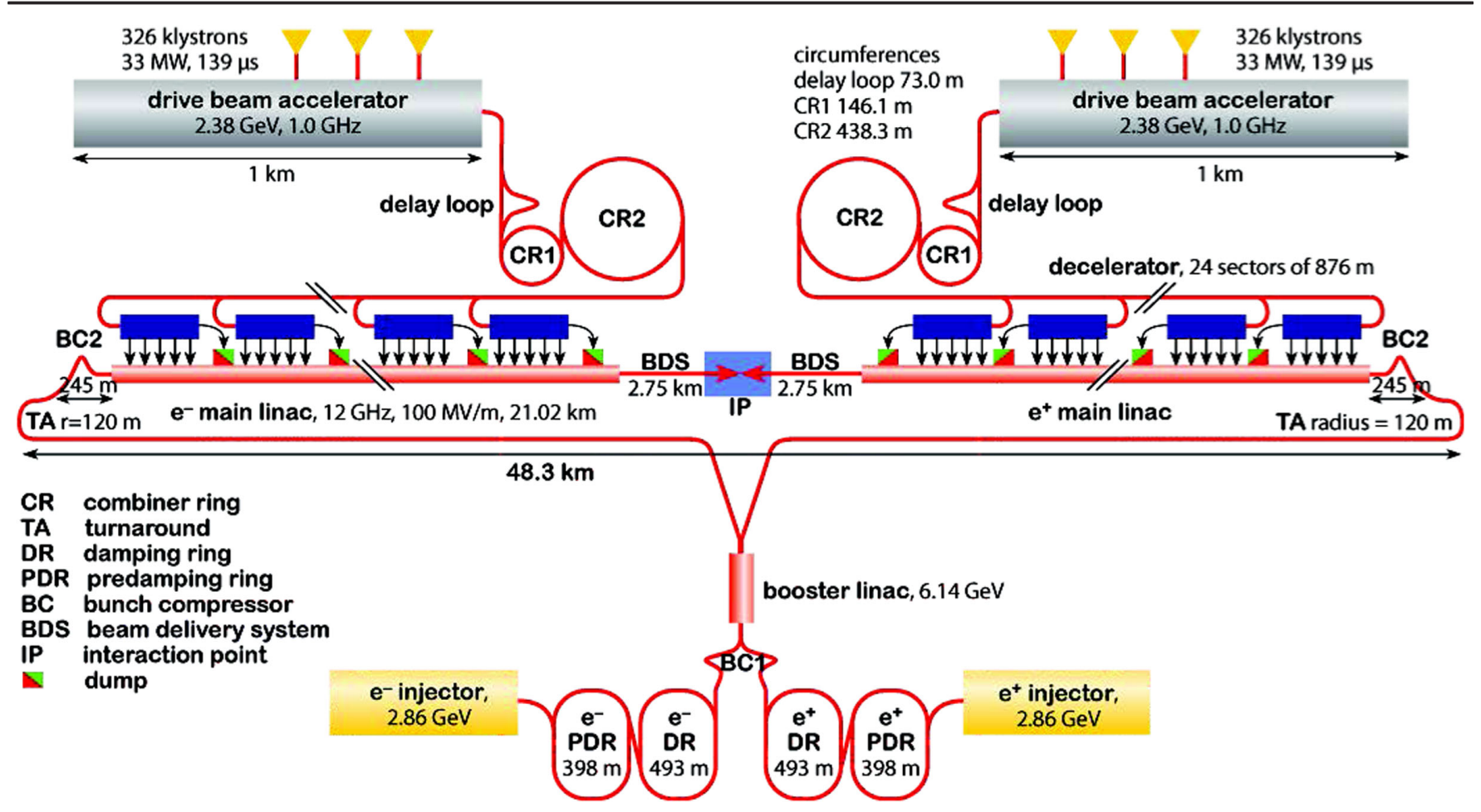

FIG. 1. Layout of the Compact Linear Collider at $3 \mathrm{TeV}$ [1] showing the combiner rings (CR) of the drive beams, the bunch compressor (BC), predamping and damping rings (PDR and DR, respectively) as well as turn/around loops (TA) and beam delivery system (BDS) of the main beams.

beam energy errors and subsequently to luminosity loss. Hence the drive beam stability is a critical aspect of CLIC feasibility.

When defining the error tolerances for the drive beam one has to consider the effects introduced by the recombination scheme. The drive beam is produced using a conventional rf source at a bunch frequency of about $0.5 \mathrm{GHz}$. After acceleration the bunch frequency is multiplied by a factor of 2 in a delay line and by factors of 3 and 4 in the two following combiner rings (Fig. 1). Hence when the drive beam reaches the $0.213 \mathrm{~m}$ long power extraction and transfer structures (PETS) in the decelerator sectors, it

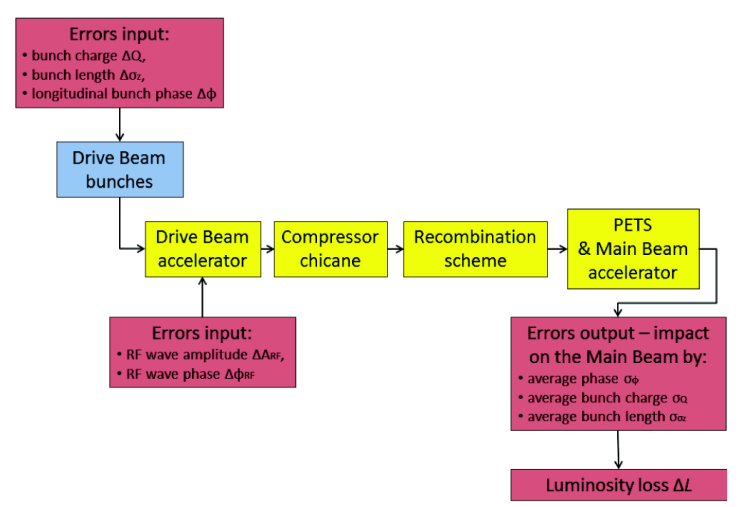

FIG. 2. Simplified process diagram of operation of drive beam error tracking simulation tool. has a bunch frequency of $0.5 \mathrm{GHz} \times 2 \times 3 \times 4=12 \mathrm{GHz}$, a bunch train length of $\mathrm{c} \times 244 \mathrm{~ns}=73.2 \mathrm{~m}$, and it provides an accelerating power of $68 \mathrm{MW}$ per beam [6].

A relative phase error of $0.2^{\circ}$ at $12 \mathrm{GHz}$ between the drive and main beams will cause a luminosity loss of $1 \%$ if the error is constant along the 24 decelerator sectors. This error is referred to as a coherent phase error $\left(\sigma_{\phi, \mathrm{coh}}\right)$. An equal luminosity loss can be caused by a random error between the decelerator sectors with an rms of $0.8^{\circ}$, which is referred to as an incoherent phase error $\left(\sigma_{\phi, \text { inc }}\right)$. Errors in the other parameters transform into bunch length and phase errors at the compressor chicane (see Sec. II B) of the drive beam and hence also ultimately lead to luminosity loss.

Equation (1) shows how the errors in the bunch charge $\left(\sigma_{Q}\right)$, length $\left(\sigma_{l}\right)$ and phase $\left(\sigma_{\phi}\right)$ of the drive beam contribute quadratically to CLIC's luminosity loss [7-9]:

$$
\begin{aligned}
\frac{\Delta \mathcal{L}}{\mathcal{L}} \approx & 0.01 \times\left[\left(\frac{\sigma_{\phi, \mathrm{coh}}}{0.2^{\circ}}\right)^{2}+\left(\frac{\sigma_{\phi, \text { inc }}}{0.8^{\circ}}\right)^{2}+\left(\frac{\sigma_{Q, \mathrm{coh}}}{0.75 \times 10^{-3} I}\right)^{2}\right. \\
& +\left(\frac{\sigma_{Q, \text { inc }}}{2.2 \times 10^{-3} I}\right)^{2}+\left(\frac{\sigma_{l, \mathrm{coh}}}{1.1 \times 10^{-2} \sigma_{z}}\right)^{2} \\
& \left.+\left(\frac{\sigma_{l, \mathrm{inc}}}{3.3 \times 10^{-2} \sigma_{z}}\right)^{2}\right]
\end{aligned}
$$

where $I$ indicates the nominal beam current and $\sigma_{z}$ the nominal bunch length. 
TABLE I. List of CLIC drive beam tolerances for coherent errors causing $1 \%$ luminosity loss $[4,10]$. The primary errors (italic) contribute directly to luminosity loss [see Eq. (1)]. Other errors lead to luminosity loss by inducing the primary errors.

\begin{tabular}{lc}
\hline \hline Parameter & Tolerance \\
\hline Bunch phase $\sigma_{\phi}$ (before feed-forward) & $2.5^{\circ}$ at $12 \mathrm{GHz}$ \\
Bunch phase $\sigma_{\phi}$ (after feed-forward) & $0.2^{\circ}$ at $12 \mathrm{GHz}$ \\
Bunch energy error $\sigma_{E}$ & $1 \%$ \\
Bunch charge error $\sigma_{Q, \text { bunch }}$ & $0.075 \%$ \\
Bunch length error $\sigma_{l}$ & $1 \%$ \\
Linac gradient error $\sigma_{A, \text { linac }}$ & $0.2 \%$ \\
Linac phase error $\sigma_{\phi, \text { linac }}$ & $0.05^{\circ}$ at $1 \mathrm{GHz}$ \\
\hline \hline
\end{tabular}

A summary of the tolerances for the coherent CLIC drive beam errors is presented in Table I. The stated tolerances for linac rf gradient and phase result from the drive beam bunch energy and phase error tolerances.

The drive beam bunch error $\sigma_{Q}$ leads to a luminosity loss via two channels: directly impacting the main beam rf loading, as well as via its impact on the drive beam phase error in the compressor chicane (as explained in Sec. II B). The stated tolerance limit results from the combined bunch error impact via both of these channels.

The tolerances are used as input parameters for the error tracking, their impact on the main beam is estimated using the realistic beam loading functions for the drive and main beam accelerating structures. Also, the study examines the possibility of the drive beam phase error reduction with the help of a feed-forward system by a factor of 12.5 , which is required to satisfy the stated tolerances.

\section{ERROR PROPAGATION THROUGH THE DRIVE BEAM}

There are four sections of the drive beam complex relevant for the analysis of error propagation (see Fig. 1): the drive beam accelerator, the compressor chicane positioned in the accelerator at a beam energy of $300 \mathrm{MeV}$, the recombination scheme consisting of the delay loop and two combiner rings and the decelerator section including the main beam accelerator modules. The influence of each of these sections on the error propagation has been included in the present analysis and the results are demonstrated in this section and in Sec. III. The analysis includes only linear effects.

\section{A. Drive beam accelerator}

The drive beam accelerator is designed to accelerate the drive beam pulses of about $140 \mu$ s length with a repetition rate of $50 \mathrm{~Hz}$ to $2.38 \mathrm{GeV}$ energy. The length of the drive beam pulse is determined by the drive beam train length needed in the decelerator ( $244 \mathrm{~ns}$ ), the recombination factor (24) and the number of drive beam decelerators (24). The bunches within the pulse have a separation of $2 \mathrm{~ns}$ and come in groups of 122 bunches; the groups are phase shifted relative to each other by $180^{\circ}$ at $0.5 \mathrm{GHz}$, as described in Sec. III A.

The acceleration is performed with klystrons via a traveling electromagnetic wave and can be described by the rf filling function - the integrated accelerating voltage in the structure induced by a delta function rf wave as a function of time. The energy absorbed by the bunches during the acceleration process is referred to as beam loading. The rf fill time, which is the time between a delta function rf pulse entering the accelerating structure and the accelerating voltage falling back to zero, has been set for the drive beam structures to $\approx 244 \mathrm{~ns}$, corresponding to the length of the compressed drive beam trains. The beam loading time of the structures has the same value. This allows partial cancellation of rf errors at a frequency corresponding to $244 \mathrm{~ns}$. It is expected that the errors will have a resonant peak at frequencies corresponding to the compressed train length and its harmonics, since these resonant errors add up constructively during the recombination process. Hence, setting the filling time of the accelerating structure to the same value as the train length will provide filtering for these critical resonant frequencies (see Sec. II A 1).

The beam loading, which is the integrated rf field absorbed by the accelerating bunch passing the structure, should be triangular. To maximize the efficiency of the acceleration, the structure is designed so that for the nominal accelerating gradient and beam current the sum of the accelerating rf field and the beam loading are zero at the downstream end of the structure.

\section{Filtering by the ideal rf filling function}

An ideal rf filling function has a constant amplitude over the filling time. The result of the convolution of an error in the amplitude or phase of the accelerating rf wave with the drive beam bunch energy error function is presented in Fig. 3. The error amplitude has been normalized to one for a

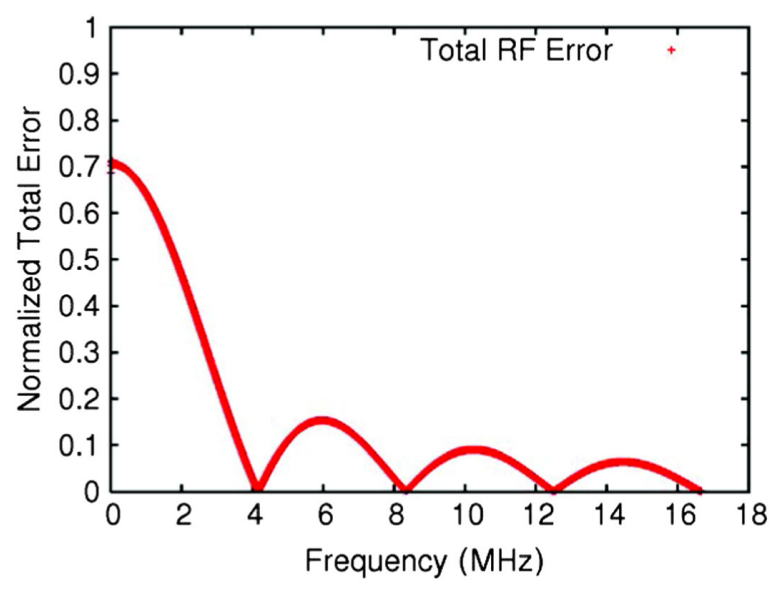

FIG. 3. Filtering factor of the ideal $\mathrm{rf}$ filling function vs $\mathrm{rf}$ phase jitter frequency. 
constant offset in amplitude or phase of the accelerating rf wave. The dips in the figure imply that if the wavelength of the error function is equal to the length of the rf wave, the signal is filtered out. In Fig. 3 and the following, the analyzed frequency range is between $50 \mathrm{~Hz}$, corresponding to the CLIC main beam train repetition frequency, and 16.67 MHz, corresponding to the center of the fourth resonant peak/dip.

\section{Filtering by ideal beam loading function}

The ideal beam loading function is triangular, and hence the filtering of the critical frequencies cannot be as effective as with a rectangular function. Figure 4 represents the filtering factor of the beam loading function for the drive beam accelerating structures.

\section{Filtering by realistic rf filling and beam loading functions}

Simulations of the rf wave and bunch propagation within the drive beam accelerating structures [2] show that the rf filling and the beam loading functions of the CLIC drive beam accelerator modules deviate to some extent from the ideal case (see Fig. 5). The calculations have been performed in the frequency domain and then transformed into the time domain using a fast Fourier transform. Higher order resonances are also included into the calculation. The nominal rf power of $15 \mathrm{MW}$ and a nominal bunch charge of $8.4 \mathrm{nC}$ have been assumed in the simulation. The amplitudes of the rf potential and of beam loading are calibrated so that the energy absorbed by the beam during acceleration, which corresponds to the cumulative beam loading function of all bunches integrated over the structure length, is half of the value of the energy induced by the rf wave, which corresponds to the integrated rf filling function. Because of this the klystrons are said to operate in the fully loaded mode. The resolution of the simulation in the frequency domain is limited; as a consequence the results display a strong oscillation of the potential functions, in particular of the rf potential function, which is unphysical.



FIG. 4. Filtering factor of the ideal beam loading function vs bunch charge jitter frequency.

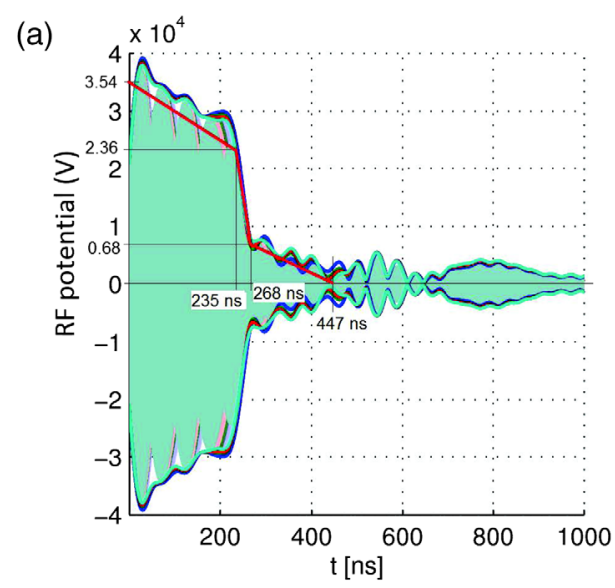

(b)

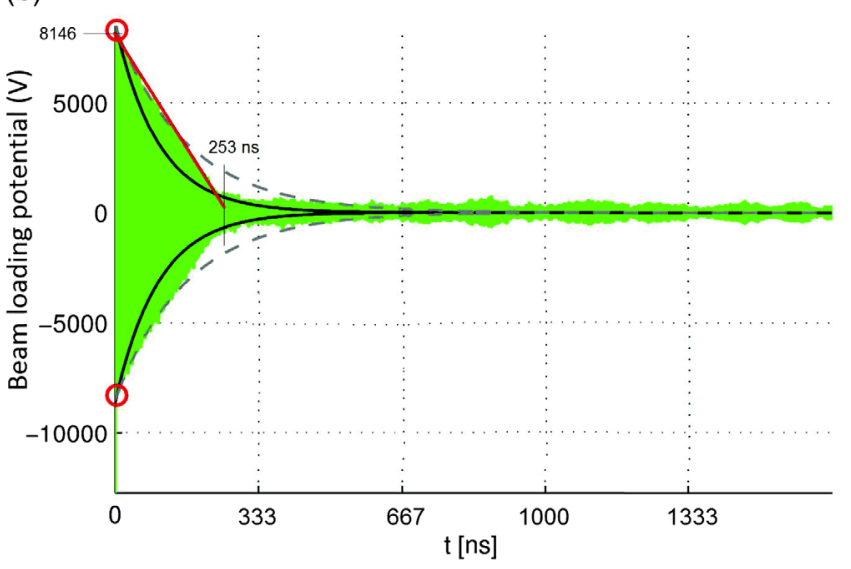

FIG. 5. Simulation results for $\mathrm{rf}$ filling potential (a) and beam loading amplitude (b) as functions of time for the CLIC drive beam cavities [2]. The approximations with linear functions are indicated by the solid red lines. In (b) two exponential approximations are indicated by the black solid and dashed lines.

Using the rf potential function [Fig. 5(a)] and the beam loading function [Fig. 5(b)] instead of the ideal functions allows the rf filtering calculation to be much more realistic. Because of the unphysical oscillations, however, an approximation with the linear functions is necessary. This approximation also makes the calculation process significantly more efficient.

\section{B. Compressor chicane}

The compressor chicane in the drive beam linac (Fig. 6) will reduce the bunch length of the drive beam from $3 \mathrm{~mm}$ to $1 \mathrm{~mm}$ at an energy of $300 \mathrm{MeV}$.

Different designs of the chicane exist. The optimal chicane has an $R_{56}$ value of $-0.1 \mathrm{~m}$, since this design allows the loosest tolerances for the beam energy and charge errors (see [4] for more details).

In the compressor chicane instabilities in several parameters cause errors in the other parameters. Their interdependences have been implemented into the software tool for drive beam error tracking. They have been modeled in a first order approximation as linear parameter 


\begin{tabular}{ccc}
\hline DBL1 & $\begin{array}{c}\text { Bunch } \\
\text { Compressor }\end{array}$ & DBL2 \\
& & \\
Beam energy & Compression energy \\
$50 \rightarrow 300 \mathrm{MeV}$ & $300 \rightarrow 2400 \mathrm{MeV}$ \\
$\begin{array}{c}\text { Bunch length: } \\
3 \mathrm{~mm}\end{array}$ & $\begin{array}{c}\text { Comm } \rightarrow 1 \mathrm{~mm} \\
\text { Bunch length: } \\
1 \mathrm{~mm}\end{array}$ \\
\hline
\end{tabular}

FIG. 6. Compressor chicane position in the drive beam linac [4].

transformations summarized in Table II. The table demonstrates how the bunches entering the drive beam linac and the compressor chicane with an error in a particular parameter (columns) leave the chicane with additional errors in other parameters (rows). For example, a bunch entering the linac with an energy error of $1 \%$ would have at the chicane entry a relative energy error of $0.17 \%$, since the energy of the beam is increased by a factor of 6 from 50 to $300 \mathrm{MeV}$ in the first section of the drive beam linac. Considering the bunch compression factor of $-0.1 \mathrm{~m}$ the additional phase error of the beam at the exit of the chicane would be

$$
\begin{aligned}
\Delta \phi_{\text {beam }(\text { outg })} & =\left(\Delta E_{\text {lina centry }} / 6\right) \times R_{56} \\
& =0.17 \% \times(-0.1 \mathrm{~m}) \\
& =-170 \mu \mathrm{m}\left(=2.5^{\circ} \text { at } 12 \mathrm{GHz}\right) .
\end{aligned}
$$

In the table $\Delta E$ is the offset of the average energy while $\delta \sigma_{E}$ is the change in the energy spread after the beam passes the chicane. The results of the impact of drive beam accelerator errors ( $\mathrm{rf}$ phase error and $\mathrm{rf}$ amplitude error) in the chicane are also considered in the calculation.

The beam energy error contributes to additional phase error in the chicane. This sets the upper limit on the energy error to $1 \%$ considering the phase error tolerance to be $2.5^{\circ}$ at $12 \mathrm{GHz}$. Also the bunch length variation tolerance of $1 \%$ dictates a single bunch charge tolerance of $1 \%$. However this is valid only for an incoherent bunch-by-bunch error along the beam. The coherent bunch charge error must be below $0.075 \%$, as dictated by the main beam accelerator $\mathrm{rf}$ amplitude tolerance [see Eq. (1)] [7].

\section{ERROR PROPAGATION THROUGH THE DRIVE BEAM RECOMBINATION SCHEME, PETS AND MAIN BEAM LINAC}

\section{A. Recombination scheme}

The frequency multiplication is designed in the following way: the bunches arriving at the delay loop are gathered in $244 \mathrm{~ns}$ long trains; the adjacent trains have a relative phase shift of $180^{\circ}$ (Fig. 7). The frequency of the rf wave in the accelerating modules is thereby $1 \mathrm{GHz}$, so that all bunches are accelerated equally. The rf deflector at the injection point of the delay loop has a frequency of $0.5 \mathrm{GHz}$, so that only bunches of every second train are led into the delay loop. The length of the delay loop is set to $244 \mathrm{~ns}$, so that the delayed train comes out of the loop simultaneously with the next train passing by the rf deflector. As a result, both trains leave the rf deflector simultaneously, their bunches being phase shifted by $180^{\circ}$. Hence, 244 ns long combined trains with 244 ns long gaps between them are created, with a bunch frequency of $1 \mathrm{GHz}$ within the train. A similar principle is used in the combiner rings, with phase shifts of $120^{\circ}$ for the 3 -combiner ring and $90^{\circ}$ for the 4-combiner ring.

The sequence of beam extractions from the first combiner ring (CR1) is nontrivial. As stated above, in the first combiner ring the beam must be combined by a factor 3 and extracted in regular intervals of $6 \times 244 \mathrm{~ns}$ for the proper operation of the second combiner ring (CR2). In order to satisfy both of these conditions, the $1 \mathrm{GHz}$ train arriving second at CR1 must be extracted without recombination, as shown in Fig. 8. In this figure, the $1 \mathrm{GHz}$ trains are represented by colored points. They are injected from the left, circulate clockwise in the ring and are extracted to the right. There is a $2 \times 244 \mathrm{~ns}$ interval passing between each step; the circumference of the ring is $4 \times 244 \mathrm{~ns}$, so each step signifies one half of a turn in the ring. The trains on the left are injected once per step and are located $244 \mathrm{~ns}$ from the injection, so that they are recombined during the injection process with the trains circulating in the ring. Doublets and triplets of points represent the trains with a combination factor of 2 and 3 , and hence with bunch frequency of 2 and $3 \mathrm{GHz}$, respectively.

Figure 9 demonstrates the $z$-position of the drive beam trains after passing the complete recombination scheme as a

TABLE II. Matrix of CLIC drive beam parameter interdependences in linear approximation [4,11]. The errors in the parameters of incoming bunches (columns) contribute in the compressor chicane to the errors in the parameters of the outgoing bunches (rows). The linac tolerances are stated for the linac section before the bunch compressor.

\begin{tabular}{lcccccc}
\hline \hline & $\begin{array}{c}\Delta \mathrm{E} \\
{[\%]}\end{array}$ & $\begin{array}{c}\Delta \sigma_{z} \\
{[\mathrm{~mm}]}\end{array}$ & $\begin{array}{c}\Delta Q_{\text {bunch }} \\
{[\%]}\end{array}$ & $\begin{array}{c}\Delta \phi_{\text {beam }} \text { [deg@1 GHz] } \\
\text { (incoming) }\end{array}$ & $\begin{array}{c}\Delta \phi_{\text {linac }} \\
\text { [deg@ 1 GHz] }\end{array}$ & $\begin{array}{c}\Delta A_{\text {linac }} \\
{[\%]}\end{array}$ \\
\hline$\delta \sigma_{E}[\%]$ & 0 & 0 & 0.005 & 0 & 0.06 & -0.018 \\
$\Delta \sigma_{z}[\mathrm{~mm}]$ & 0 & 1 & 0.01 & 0.09 & -0.09 & -0.024 \\
$\Delta Q_{\text {bunch }[\%]}[\mathrm{outg})[\mu \mathrm{m}]$ & 0 & 0 & 1 & 0 & 0 & 0 \\
$\Delta \phi_{\text {beam }}(\mathrm{outg})$ & 80 & 750 & -850 \\
\hline \hline
\end{tabular}




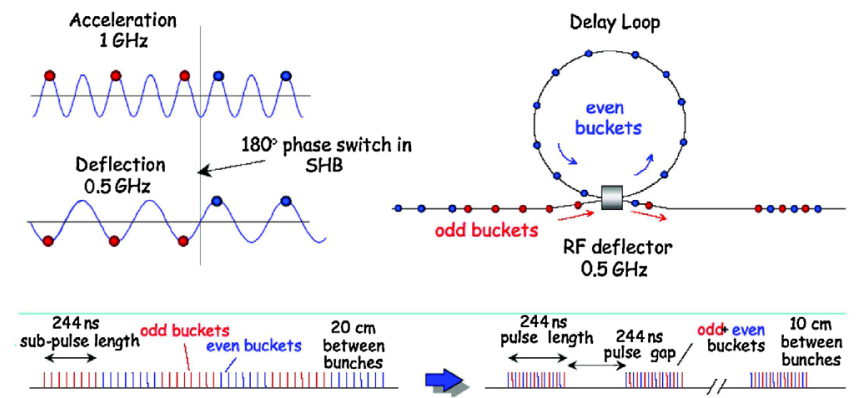

FIG. 7. Principle of operation of the CLIC recombination scheme [12].

function of their $z$-position before the recombination. Due to the zoom factor the singular bunches are not distinguishable, however one can recognize that the 24 trains are overlapped and occupy the same $244 \mathrm{~ns}$ long intervals in $z$-position. The bunch frequency within this interval is $24 \times 0.5 \mathrm{GHz}=$ $12 \mathrm{GHz}$ and the intervals are separated by $23 \times 244 \mathrm{~ns}=$ $5612 \mathrm{~ns}$ long gaps. The train with the lowest $z$-position after passing the recombination scheme is the $1 \mathrm{GHz}$ train extracted from the first combiner ring without recombining with other trains. It is not used for the main beam acceleration, in contrast to the following $12 \mathrm{GHz}$ trains.

\section{B. Power extraction and transfer structures and main beam linac}

After the calculation of the drive beam errors propagating through the drive beam linac, passing the chicane and being recombined in the drive beam recombination scheme, it is necessary to calculate the impact of these errors on the main beam. We consider the ideal (error free) extraction of the rf wave from the drive beam, since the extraction errors are expected to be negligibly small and the broader bandwidth of the PETS structures allows the response function to be treated assuming the ideal case. After extraction, the rf wave function which includes the errors from the drive beam is folded with the main beam $\mathrm{rf}$
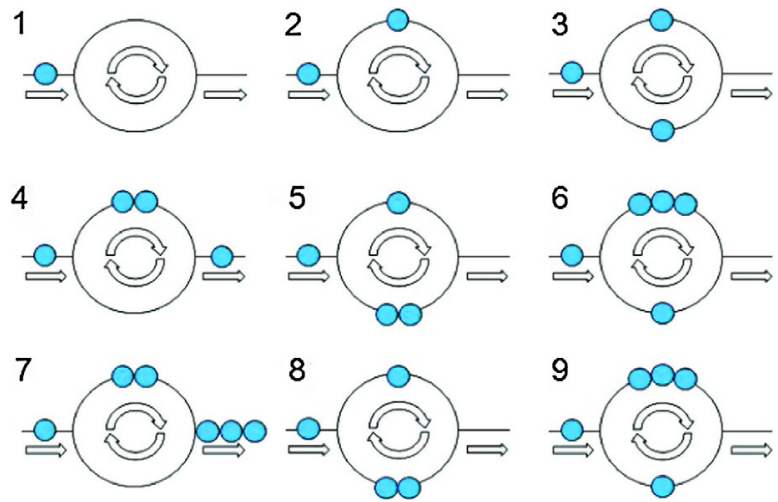

FIG. 8. Scheme of first several circulations in the first combiner ring. In step 4 the $1 \mathrm{GHz}$ train is extracted without recombination [5].

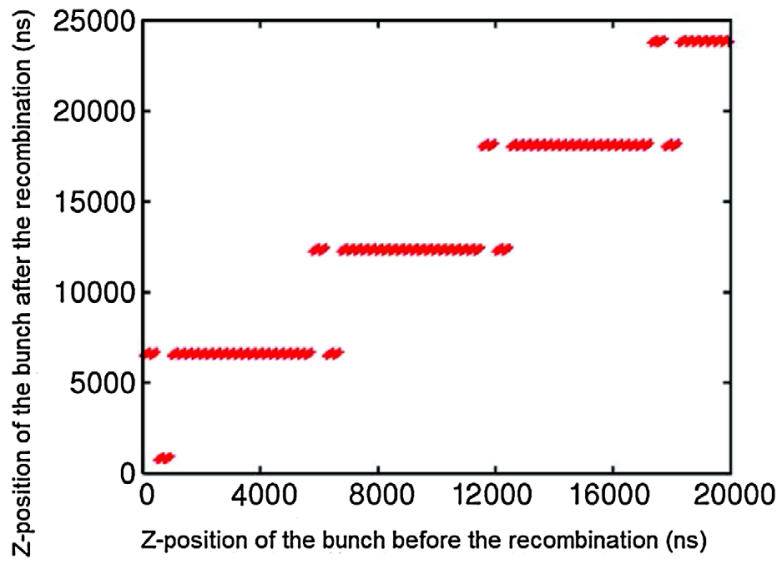

FIG. 9. Bunch $z$-position after passing the drive beam recombination scheme vs bunch $z$-position before.

potential [Fig. 10(a)] and beam loading [Fig. 10(b)] functions, which yields the impact of the drive beam bunch charge and phase errors on the main beam acceleration.

The rf potential and beam loading are the results of a simulation performed in the frequency domain around the resonant peak in the interval from 11.7 to $12.3 \mathrm{GHz}$ [3].

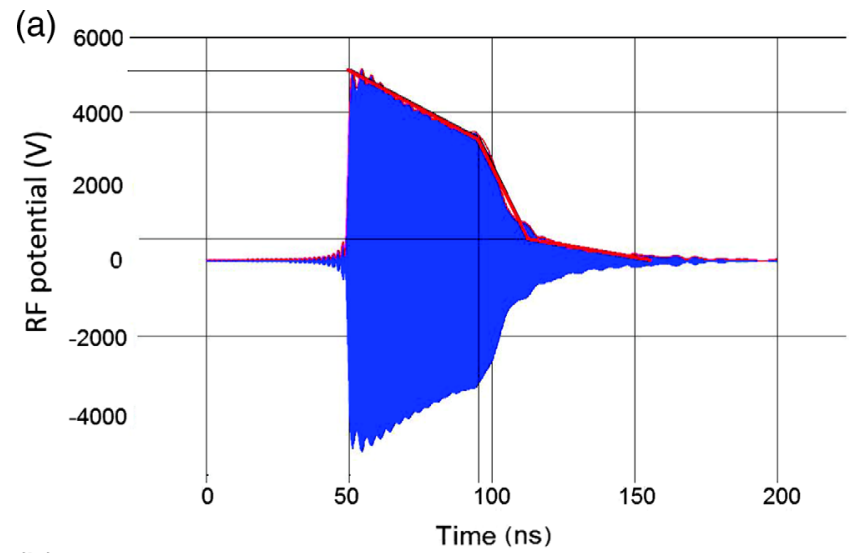

(b)

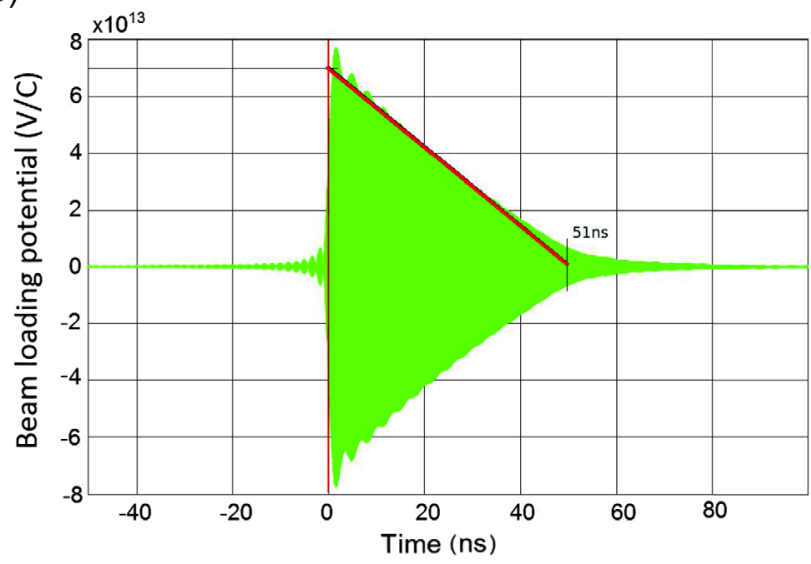

FIG. 10. Simulation result for rf filling potential (a) and beam loading amplitude (b) as a function of time for CLIC main beam accelerating structures [3]. Approximations by linear functions are indicated by the red lines. 


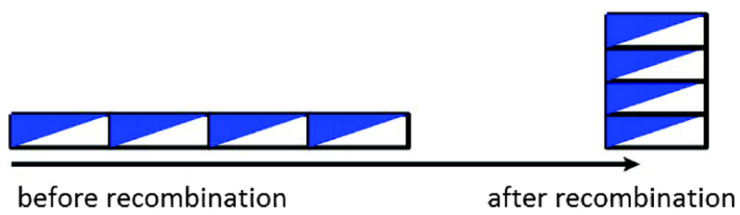

FIG. 11. Depiction of train errors' overlap. The blocks on the left and right represent the trains before and after the recombination, respectively. After the recombination the error functions of the trains overlap.

\section{Filtering by the recombination scheme and main beam accelerating structures}

Despite the fact that the recombination scheme does not impact the errors of the drive beam bunches, the recombination introduces a significant filtering effect. This occurs in the following way: when the trains are recombined in the delay line and in the combiner rings, the errors from different trains come together in one train (Fig. 11) and the error functions overlap.

In the case when the wavelength of the error jitter is the same as the train length, the errors add up constructively (see the peaks at the frequencies $4.17 \mathrm{MHz} \times n$ in Fig. 12). However, if the wavelength of the error jitter is not in resonance with the train length, errors of the bunches positioned next to each other cancel out on the time scale of several tens of nanoseconds, which is the time scale of $\mathrm{rf}$ filling in the main beam accelerating structures. Consequently, these errors have a significantly lower impact on the main beam and effectively are filtered by the recombination scheme.

Multiplying the filtering factor for the ideal rf filling function calculated in Sec. II A 1 by the phase error yields the total error shown in Fig. 13. The distribution is valid for both drive beam $\mathrm{rf}$ phase and drive beam rf amplitude errors. One can recognize that the peaks present in Fig. 12 are filtered out, since the filling time of the accelerating

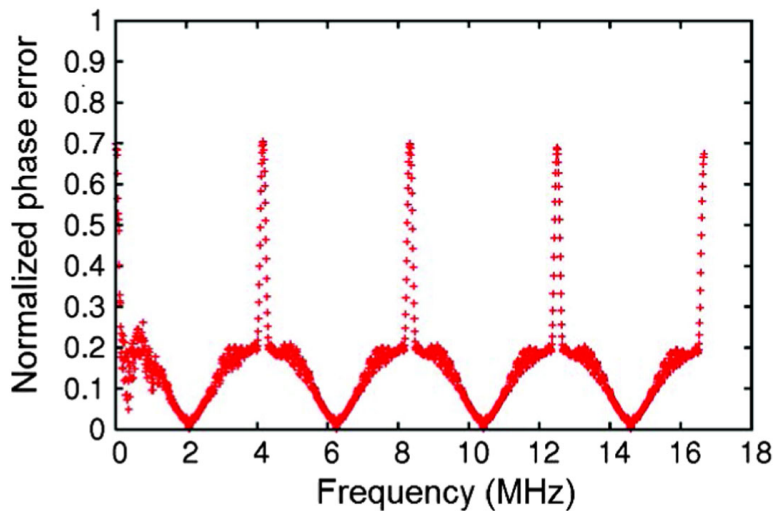

FIG. 12. Normalized phase error amplitude vs jitter frequency after filtering by recombination scheme and main beam acceleration structures. The incoming phase error has a white noise distribution with amplitude normalized to unity.

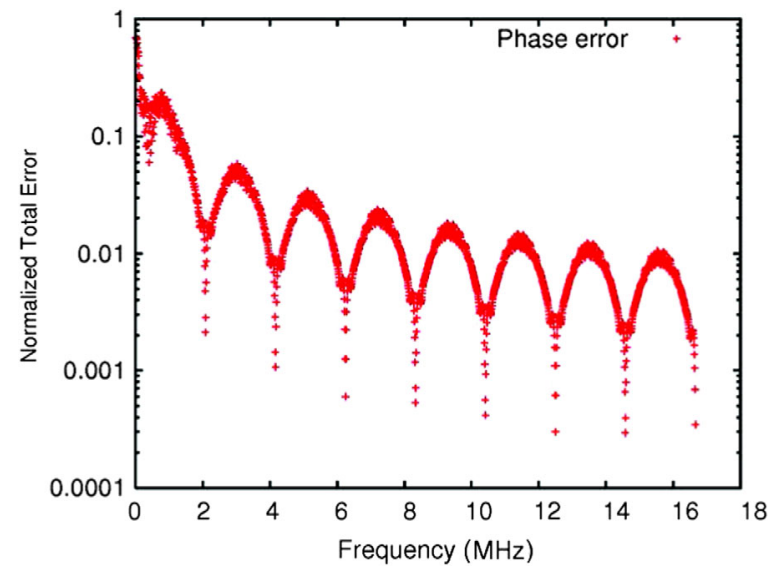

FIG. 13. Normalized phase error after filtering by the recombination scheme and the ideal drive beam rf filling function vs $\mathrm{rf}$ phase jitter frequency.

drive beam rf wave has the same value as the drive beam train length.

The convolution of the recombination scheme error filtering distribution with the ideal beam loading filtering distribution (Sec. II A 2, Fig. 4) is presented in Fig. 14. The resonant peaks of the phase error remain after filtering with the linearly decreasing beam loading function.

Convolution of the realistic drive beam rf filling and beam loading functions presented in Sec. II A 3 with the drive beam error function leads to the results shown in Figs. 15 and 16. In Fig. 15 the phase error is caused by a $0.1 \%$ error in bunch charge, which leads to an error in beam loading and ultimately to the phase error. Figure 16 demonstrates the phase error caused by a $0.05 \%$ drive beam accelerating structure rf amplitude error. The distribution would have the same form for the drive beam $\mathrm{rf}$ phase jitter. The realistic beam loading function [Fig. 5(b)] has an approximately triangular form, and so the filtering of

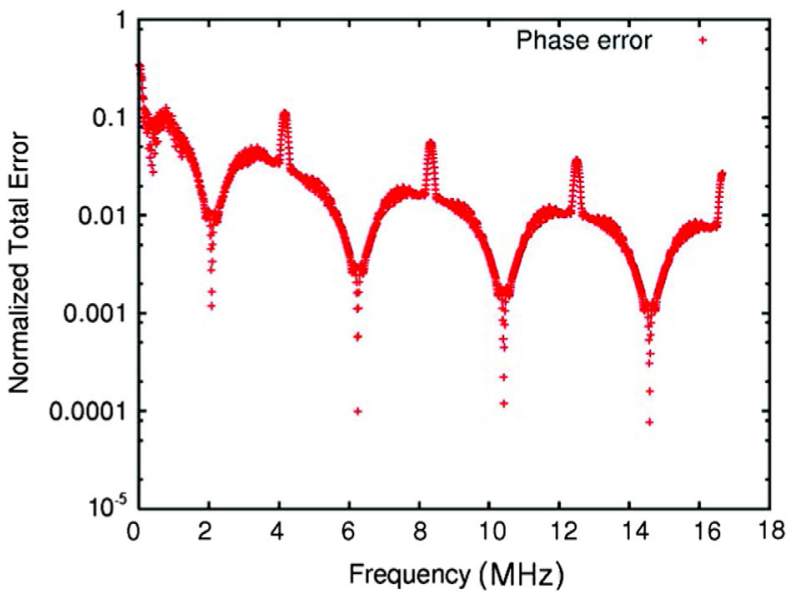

FIG. 14. Phase error, caused by the white noise jitter of the bunch charge, after the filtering by the recombination scheme and the ideal drive beam loading function vs bunch charge jitter frequency. 


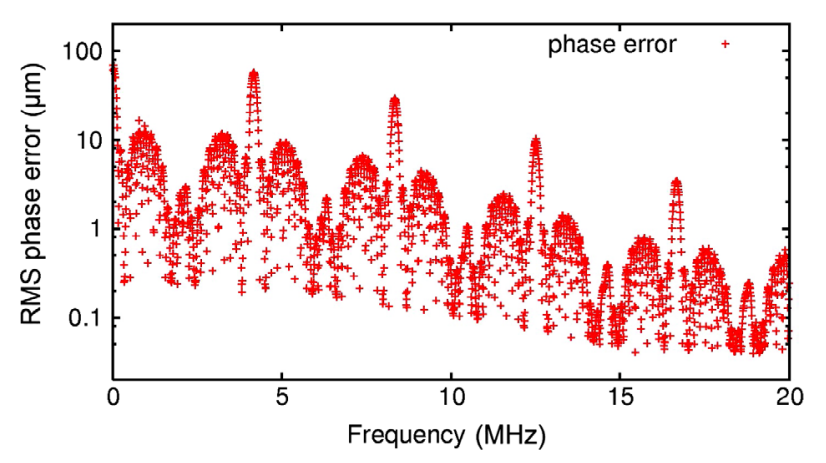

FIG. 15. Phase error caused by $0.1 \%$ drive beam bunch charge error jitter vs frequency of the bunch charge jitter, calculated with realistic rf filling and beam loading functions. The maximal phase error value of $170 \mu \mathrm{m}$ correspond to $2.5^{\circ}$ at $12 \mathrm{GHz}$.

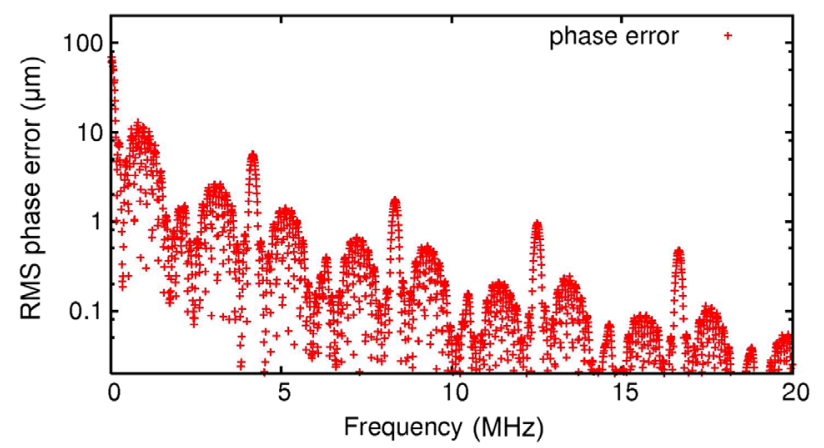

FIG. 16. Phase error caused by $0.05 \%$ drive beam rf amplitude jitter vs frequency of the rf amplitude jitter, calculated with realistic rf filling and beam loading functions. The maximal phase error value of $170 \mu \mathrm{m}$ correspond to $2.5^{\circ}$ at $12 \mathrm{GHz}$.

the errors, in particular of the resonant peak frequencies, is not as effective as for the rf filling function, which is nearer to a rectangular form [Fig. 5(a)]. Consequently, the high-frequency filtering for the phase error caused by the drive beam rf amplitude error is more effective than for the phase error coming from the bunch charge error (compare Figs. 15 and 16).

\section{BUNCH CHARGE FEEDBACK AND PHASE FEED-FORWARD SYSTEMS}

\section{A. Drive beam bunch charge feedback system}

As it has been mentioned in Sec. I A the bunch charge error has two ways of affecting CLIC's luminosity. First, it has a direct impact on the main beam accelerating rf amplitude. Additionally, it causes the beam phase error in the drive beam linac and in the compression chicane, which later affects the main beam rf filling.

For the maximal allowed luminosity loss of $1 \%$ the bunch charge stability specification for the coherent errors is $0.075 \%$, when considering both luminosity loss channels [7]. The stability achieved at the PHIN photoinjector at CERN, which is in development for CLIC, is $0.25 \%$ [13].
Hence, an additional stabilization is required, which can be performed by a fast feedback system. This system would measure the bunch charge (averaged over the monitor's bandwidth), amplify the measurement input (averaging it over the amplifier bandwidth) and send it as a correction signal to the electron gun within one drive beam pulse.

The feedback system has two timing parameters that can be optimized-the latency and the gain duration. The latency is the time between the bunches passing the bunch charge measurement system and the start of correction application. The gain duration is the time between the start of correction application and the application of the full correction, which corresponds to the ramping time of the amplifier for the correction kicker. A linear rise of the signal during the gain duration has been measured at the FONT amplifiers [14] and hence was assumed for this simulation.

The variation spectrum of these two parameters is presented in Fig. 17. For this study, a white noise between zero frequency and $20 \mathrm{MHz}$ was assumed. The $20 \mathrm{MHz}$ upper limit has been selected because it corresponds to an rf fill time of the main beam accelerating structures (about $50 \mathrm{~ns}$ ) and hence the errors above this frequency are filtered out sufficiently by the convolution with the main beam rf filling function. Also, as has been demonstrated in the previous section, the recombination scheme error filtering is very effective for the high frequency jitter and hence this jitter has a small contribution to the overall phase error of the main beam.

The best values for the correction efficiency are reached when the sum of latency time and gain duration have values around $n \times 244 \mathrm{~ns}(n \in \mathbb{N})$, corresponding to the length of the recombined trains. If the bunch charge correction happens after approximately this time, the bunches with the corrected values are positioned next to the bunches at which the errors were measured. Hence the errors will cancel each other out on a time scale of the rf filling.

The distribution shows that the error at a high gain duration time is relatively low, independent of the feedback latency. The reason for it is that the correction with a high

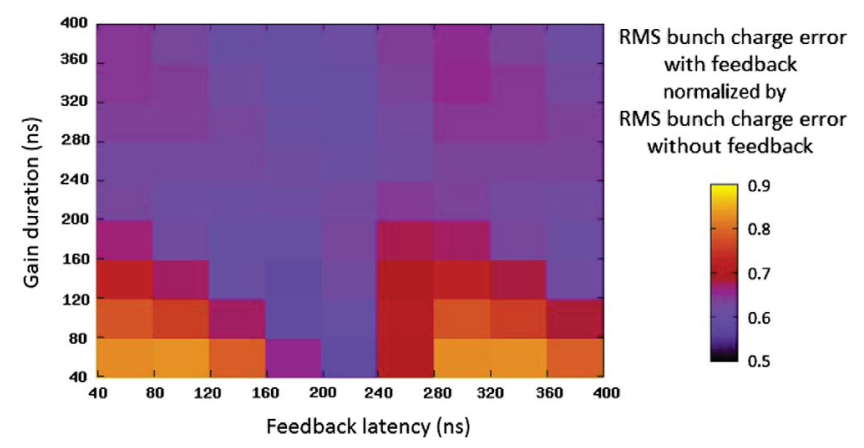

FIG. 17. Normalized total bunch charge error as a function of gain duration and latency time of the charge feedback system. A normalized white noise before the recombination scheme filtering and the feedback application is assumed. 
gain duration is effective for the filtering of the low frequency errors. At the same time, the effect of the correction being destabilizing for feedback latency times of $(n+1 / 2) \times 244 \mathrm{~ns}$ is decreased by the prolonged application time of the correction signal.

The weak absolute minimum of the distribution is at a latency time of $244 \mathrm{~ns}$ and a gain duration time of $0 \mathrm{~ns}$. However, such a feedback bandwidth is not possible to achieve. A feedback system with an achievable gain duration time of $40 \mathrm{~ns}$ (corresponding to a bandwidth of $8.75 \mathrm{MHz}$ ) and a latency time of $200 \mathrm{~ns}$ can improve the phase stability by $45 \%$. However, the improvement is insufficient for the stabilization requirement defined above: the bunch charge stability of $0.25 \%$ achieved at the PHIN experiment would need a reduction by a factor of 3.3 in order to satisfy the CLIC stability requirement of $0.075 \%$. Hence, additional bunch charge stabilization is needed if a PHIN-like injector is to be used as the CLIC drive beam source. However, in order to reduce the beam phase error caused by the bunch charge errors, a phase feed-forward system can be used (see Sec. IV B).

\section{B. Drive beam phase feed-forward system}

A feed-forward system is similar to a feedback system, with the exception that the signal with the measurement data reaches the correction point before the beam does. In case of the CLIC drive beam this means that the signal of the drive beam phase measurement is sent directly to the phase correction chicane, while the beam propagates through a longer turnaround (see Fig. 18 [10]).

This is possible if the system is installed at the turnaround sections of the beam line, e.g., it is planned to position the phase monitors before the final turnaround of the drive beam and a phase correction chicane directly after it. The phase signal from the monitors would be amplified and sent to the chicane of electromagnetic kickers, which would modify the trajectory (and hence the time of flight) of the bunches and so correct their longitudinal position.

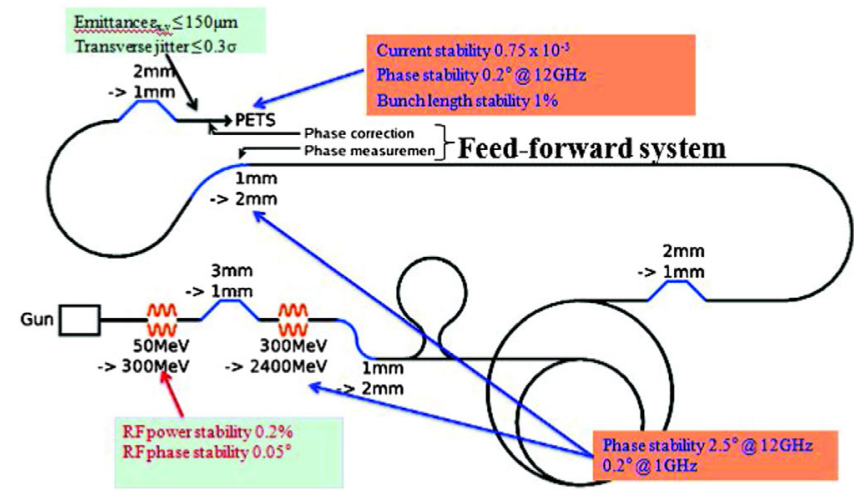

FIG. 18. Drive beam layout with indicated position of the feedforward system and the tolerances for various drive beam parameters along the beam.
The feed-forward system has a limited bandwidth and a linear rise of the amplifier signal during the gain duration. Since the starting point of correction application can be set before the arrival of the beam at the correction module, the feed-forward system can be simulated in the same way as a feedback system, but with a negative latency time. In fact, the best correction efficiency can be achieved if the latency time $t_{l}$ is set to minus half of gain duration $t_{\text {gain }}, t_{l}=-\frac{1}{2} t_{\text {gain }}$, since with that setting the bunch with the measured signal is positioned in the center of the correction.

Figure 19 demonstrates the effect of feed-forward systems with different amplifier bandwidths on the phase error. The red distribution represents the phase error of incoming white noise in drive beam bunch phase with amplitude of $175 \mu \mathrm{m}$ (corresponding to $2.5^{\circ}$ at $12 \mathrm{GHz}$ ) after the recombination scheme error filtering. The green and blue distributions show this phase error after the application of a feed-forward correction with 50 and $10 \mathrm{~ns}$ gain duration, respectively.

The horizontal red line corresponds to the tolerance of $14 \mu \mathrm{m}$, which has to be satisfied across the complete frequency spectrum. The figure illustrates that the highfrequency errors $(\geq 6 \mathrm{MHz})$, apart from the frequencies resonant to the train length, are filtered well by the recombination scheme and by the convolution with the drive beam and main beam rf functions. However, the lowfrequency errors still need to be corrected in order to satisfy the overall tolerance. On the other hand, the feed-forward significantly improves the phase stability, in particular in the low-frequency part of the spectrum. For instance, a feed-forward system with a realistic gain duration of $50 \mathrm{~ns}$ improves the phase error between zero frequency and $1 \mathrm{MHz}$ by a factor 50. Since the recombination scheme and convolution with drive and main beam rf structure functions is more effective in filtering the high-frequency noise, the feed-forward is an effective complementary stabilization system.

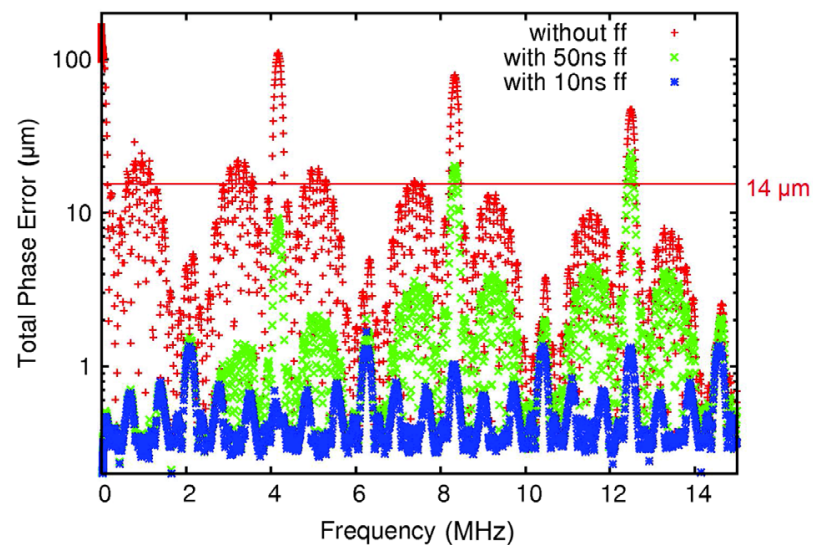

FIG. 19. Phase error as a function of jitter frequency without feed-forward and with 50 and 10 ns gain duration feed-forward. The tolerance of $14 \mu \mathrm{m}$ phase error is indicated with a horizontal line. 
The feed-forward system is designed to reduce the phase error on average by a factor of 12 , with the tolerance before the feed-forward correction being $175 \mu \mathrm{m}$ and after the correction being $14 \mu \mathrm{m}$. The efficiency of the feed-forward system strongly depends on the gain duration, with shorter gain duration being more effective at higher frequencies. Integrating the phase error amplitude $\sigma_{\phi}(f)$ quadratically (since the phase errors at different frequencies are linearly independent) over the frequency yields the figure of merit $\mathcal{A}$ for a feed-forward correction [see Eq. (3)]. The phase error amplitude is multiplied by the noise power spectrum $P(f)$ in order to obtain results not only for the white noise $[P(f)=1]$, but also for different noise spectra, e.g., for pink $[P(f) \propto 1 / f]$ and red noise $\left[P(f) \propto 1 / f^{2}\right]$ :

$$
\mathcal{A}=\sqrt{\int_{50 \mathrm{~Hz}}^{20 \mathrm{MHz}} \sigma_{\phi}^{2}(f) P(f) d f} .
$$

It is necessary to set a positive lower frequency limit for integration, since the pink and red noise spectra are divergent at zero frequency. The limit is set to $50 \mathrm{~Hz}$, since this frequency corresponds to the CLIC main beam pulse repetition rate. The wavelength of the jitter at $50 \mathrm{~Hz}$ is $c \times 20 \mathrm{~ms}$, hence the error change along the $c \times 140 \mathrm{~ns}$ long main beam is insignificant [about $\sin (140 \mathrm{~ns} / 20 \mathrm{~ms})=7 \times 10^{-6}$ of error amplitude] and so the $50 \mathrm{~Hz}$ error can be considered as almost coherent along the main beam. Also, the feed-forward system is designed to perform intrapulse phase corrections, hence the jitter frequencies below the train repetition rate are of minor importance for the stabilization simulation.

The $20 \mathrm{MHz}$ upper limit has been chosen because it corresponds to an $\mathrm{rf}$ fill time of the main beam accelerating structures (about $50 \mathrm{~ns}$ ) and hence the errors above this frequency are filtered out sufficiently by the convolution with the main beam rf filling function. Also, as demonstrated above, the recombination scheme error filtering is very effective for the high frequency jitter and hence this jitter has a small contribution to the overall phase error of the main beam and to CLIC's luminosity loss.

Figure 20 shows the result of such integration. The recombination scheme filters out the high-frequency errors more effectively, hence for the white noise the reduction of overall error due to the filtering by the recombination scheme is more significant than for the pink and red noise. The error with a constant value is not impacted by the recombination scheme at all. On the other hand, the feedforward system with lower bandwidth performs much better on the constant error as well as on red and pink noise, since it filters mostly the low frequency component. A higher bandwidth of the feed-forward system (lower gain duration) is required in order to achieve a significant level of correction for the white noise. The bandwidth requirements for the pink and red noise are more relaxed. Hence,
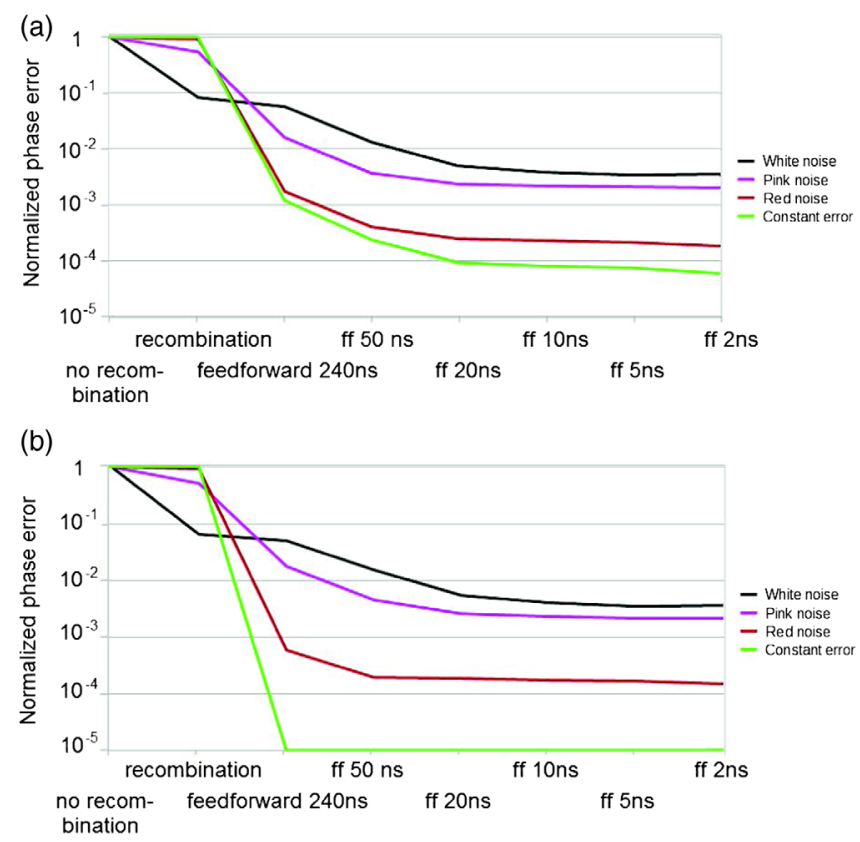

FIG. 20. Normalized phase error of the main beam accelerating rf generated by the errors in drive beam bunch charge (a) and in drive beam accelerating rf phase (b) for different noise spectra. The error value is plotted before recombination (first entry on the $x$ axis), after filtering by the recombination scheme (second entry on the $x$ axis) and after feed-forward correction with different gain durations (following entries on the $x$ axis) [15].

one must conclude that the bandwidth of the system must be selected dependent on the frequency spectrum anticipated for the phase error at the final turnaround loop.

The inverted function $1 / \mathcal{A}$ defines the tolerance improvement factor for a particular noise type. In order to achieve the required improvement factor of 12 for the constant offset, as well as for the red and pink noise, the feed-forward system with a bandwidth of $0.35 / 240 \mathrm{~ns}=$ $1.46 \mathrm{MHz}$ is fully sufficient (the bandwidth is calculated as 0.35 divided by the gain duration, according to the standard rise time definition of signal rise from $10 \%$ to $90 \%$ of its amplitude, and considering that the feed-forward amplifier gain duration is the only limiting factor for the correction rise time). However, for a correction of white noise by a factor of 12 a higher bandwidth is required. For example, the feed-forward system with a bandwidth of $0.35 / 20 \mathrm{~ns}=$ 17.5 MHz would improve the phase tolerance by a factor of $\approx 17$ for the phase errors induced by the white noise bunch charge jitter and by a factor of $\approx 12$ for the phase errors induced by the white noise jitter of the rf phase.

\section{SUMMARY AND CONCLUSIONS}

Simulation studies of the longitudinal phase stabilization of the CLIC drive beam have been performed. The propagation of drive beam errors through the drive beam accelerator, compressor chicane and the recombination scheme has been studied. The impact of the phase errors 
on the main beam and on CLIC's luminosity has been evaluated. The simulations incorporated linear effects in the chicane and the realistic drive and main beam rf filling and beam loading functions.

The analysis has shown that high-frequency errors are significantly filtered by the recombination scheme-by more than a factor 10 for the frequencies $>100 \mathrm{kHz}$, except at the resonant peaks at the frequencies $4.17 \mathrm{MHz} \times n$. Low frequency noise $(<100 \mathrm{kHz})$ is poorly filtered by the recombination scheme.

Two solutions for the stabilization of the drive beam phase have been analyzed-a feedback system for bunch charge errors which cause the phase errors, and a feedforward system which should directly correct the phase of the bunches at the final turnaround of the drive beam before the decelerators.

It has been found that a realistic feedback system with a bandwidth of $8.75 \mathrm{MHz}$ can improve the drive beam current stability by $45 \%$.

The feed-forward system analysis has demonstrated a strong potential reduction of the phase error, in particular for the low frequency noise $(\approx$ factor 50 for white noise up to $1 \mathrm{MHz}$ and realistic $50 \mathrm{~ns}$ feed-forward bandwidth). The required phase stabilization by a factor of 12 could be achieved for the red and pink noise spectra with a $1.46 \mathrm{MHz}$ feed-forward amplifier bandwidth (for errors with frequencies from $50 \mathrm{~Hz}$ to $20 \mathrm{MHz}$ ). The same improvement factor could be possible for white noise only with $17.5 \mathrm{MHz}$ amplifier bandwidth. Hence, the bandwidth requirements of the system depend on the frequency spectrum anticipated for the phase error at the final turnaround loop. The results are, however, in any case encouraging, since even the bandwidth requirement of $17.5 \mathrm{MHz}$ is realistic to fulfill. Hence, it could be confirmed that for drive beam phase error tolerance of $2.5^{\circ}$ at $12 \mathrm{GHz}$ before the feed-forward the luminosity loss of $<1 \%$ is realistic.

A prototype of a CLIC phase feed-forward system is currently under development and has been tested at the CLIC Test Facility (CTF3) in 2014 [16]. Measurements of CTF3 drive beam phase error have been performed and allow simulation of the error propagation along CTF3 in order to predict the impact of the prototype on the phase errors [17]. These predictions show that the prototype will have a measurable effect on CTF3 phase errors, allowing the experimental test of its functionality.

[1] M. Aicheler, P. Burrows, M. Draper, T. Garvey, P. Lebrun, K. Peach, N. Phinney, H. Schmickler, D. Schulte, and N. Toge CLIC-CTF3 Collaboration, A multi-TeV linear collider based on CLIC technology, CLIC Conceptual Design Report No. CERN-2012-007, 2012.

[2] R. Wegner, CLIC-Note-945 (private communication).

[3] O. Kononenko, Compensation of transient beam-loading in CLIC main linac, 2010, http://cds.cern.ch/record/ 1302739/files/CERN-ATS-2010-209.pdf.

[4] A. Aksoy, Simulations for CLIC drive beam linac, arXiv:1202.5628v1.

[5] A. Gerbershagen, CLIC drive beam phase stabilisation, Doctoral thesis, University of Oxford [Report No. CERNTHESIS-2013-160, Sec. 3.1.4, 2013 (unpublished)].

[6] CLIC parameter table, 2010, http://clic-meeting.web.cern .ch/clic-meeting/clictable2010.html.

[7] D. Schulte et al., Status of the CLIC phase and amplitude stabilisation concept, in Proceedings of the 25th International Linear Accelerator Conference, LINAC2010, Tsukuba, Japan (KEK, Tsukuba, Japan, 2010), MOP024.

[8] D. Schulte and R. Tomas, Dynamic effects in the new CLIC main linac, in Proceedings of the 23rd Particle Accelerator Conference, Vancouver, Canada, 2009 (IEEE, Piscataway, NJ, 2009).

[9] D. Schulte, E. J. N. Wilson, and F. Zimmermann, The impact of longitudinal drive beam jitter on the CLIC luminosity, CLIC Note No. 598, 2004.

[10] D. Schulte et al., CLIC phase stabilisation, for CLIC Phase Stability Meeting, 2011.

[11] A. Aksoy, Drive beam linac longitudinal tolerances, 2010, http://indico.cern.ch/event/103860/contribution/1/ material/slides/0.pdf.

[12] F. Tecker, DB generation: phase coding, CTF3 results, CLIC-ACE, 2010, http://indico.cern.ch/event/81094/ session/13/contribution/33/material/slides/1.pdf.

[13] E. Chevallay, M. Csatari, A. Dabrowski, S. Doebert, D. Egger, V. Fedosseev, O. Mete, M. Olvegaard, and M. Petrarca, PHIN photo-injector as the CLIC drive beam source, J. Phys. Conf. Ser. 347, 012036 (2012).

[14] B. Constance, Design and beam testing of a fast, digital intra-train feedback system and its potential for application at the International Linear Collider, D.Phil. thesis, University of Oxford, 2011.

[15] A. Gerbershagen et al., Simulation of phase stability at the flat top of the CLIC drive beam, in Proceedings of the 2nd International Particle Accelerator Conference, San Sebastián, Spain (EPS-AG, Spain, 2011), TUPC013.

[16] P. Skowronski, Design of phase feed forward system in CTF3 and performance of fast beam phase monitors, in Proceedings of the 4th International Particle Accelerator Conference, IPAC-2013, Shanghai, China, 2013 (JACoW, Shanghai, China, 2013), WEOBB203, pp. 2097-2099.

[17] A. Gerbershagen et al., Performance simulations of a phase stabilization system prototype for CTF3, in Proceedings of the 3rd International Particle Accelerator Conference, New Orleans, LA, 2012 (IEEE, Piscataway, NJ, 2012), WEPPP066. 